\title{
Effect of OPEFB-NCC Axial Ratio on Optical Properties of NCC Film
}

\author{
R. Rohaizu and W. D. Wanrosli
}

\begin{abstract}
In this study, we report the effect of axial ratio on the properties of films from OPEFB-NCC. OPEFB-NCC is prepared via the TEMPO oxidation process whilst NCC's with different axial ratio (designated as LAR-NCC, MAR-NCC and HAR-NCC for short, medium and long fibrils respectively) are produced using the centrifugation and filtration technique. OPEFB-NCC films are prepared using the standard film casting method. SEM micrographs show that the surface morphology of all types of films appear to be smooth and homogenous with multilayered deposition of particles, whereas the cross section morphology shows the presence of voids in the HAR-NCC film but almost none were visible in the MAR-NCC and LAR-NCC films. MAR-NCC and LAR-NCC films are visually transparent whilst the HAR-NCC films are opaque in nature which is in agreement with the light transmittance measurement where higher transmittances were observed in the LAR-NCC film as compared to MAR-NCC and HAR-NCC film in the visible wavelength section. Thermal analyses show that there are no significant differences in thermal stability even though the axial ratios of samples are different.
\end{abstract}

Index Terms-Axial ratio, OPEFB-NCC, OPEFB-NCC film, SEM, optical properties.

\section{INTRODUCTION}

In Malaysia, oil palm (Elaeis guineensis) is being cultivated as an agricultural crop to produce palm oil, which is an important feedstock to the food industry for edible oil production and also for the oleochemicals industry for the production of cosmetics, soap and others [1]. As the world's largest exporter and the second largest producer of palm oil [2], the oil palm industry has become the most important sector for the agricultural and economy of Malaysia. In addition to the obvious benefit, an estimated 70 million tonnes of oil palm biomass is generated in Malaysia annually, thus making these lignocellulosic wastes a major disposal problem [2]. These includes the oil palm fronds (OPF) and oil palm trunks (OPT) which are available at the plantation site, empty fruit bunches (EFB), palm kernel shells, mesocarp fibre and palm oil mill effluent (POME) at the mill sites.

Realizing the potential of these oil palm biomass, numerous research on their utilization have been carried

Manuscript received February 9, 2014; April 21, 2014. This work was supported in part by the Research University under grant 1001/PTEKIND/814122, 1001/PTEKIND/845027 and Minister of Higher Education Malaysia for sponsoring postgraduate studies of R. Rohaizu.

Rohaizu and W. D. Wanrosli are with the School of Industrial Technology, Universiti Sains Malaysia, 11800 Pulau Pinang, Malaysia (tel.: +604653 2354; fax: +60 4657 3678; e-mail:roh_aizu@yahoo.com, wanrosli@usm.my). over the years, examples are as alternative raw materials for biofuel production, paper making pulp, wood production and biobased chemicals [1]-[5]. In this regards, amongst the abovementioned types of oil palm biomass wastes, EFB is the most targeted residues in the market due to the availability of this material in huge quantity that can be supplied at reasonable costs. In this present paper, we investigate the use of $\mathrm{EFB}$ as a source for producing nanocellulose crystals (NCC) which has the potentials as an alternative to wood in future.

Cellulose is the most abundant biopolymer in nature, which is renewable, biodegradable, as well as non-toxic. Cellulose has attracted attention for its potential in the preparation of advanced material, one of which is conversion into nanocellulose crystal (NCC) which is of significant importance due to its ability to offer a stronger material [6]-[10]. NCC is the basic building unit of cellulose, particles in rod like shape, and size in the range of 100-400 $\mathrm{nm}$ length and less than $10 \mathrm{~nm}$ in width depending on cellulose sources [6], [10].

Presently demand of nano size material is on the increase due to the capability of these materials to meet high expectation and holds great promise of revolutionizing materials for use in the 21 st century. In their extremely small size with relatively greater surface area, nanocellulose has many interesting properties that are different from the conventional bulk material; for example, nanocellulose paper or film has been shown to produce optically transparent. This optically transparent nanocellulose film is expected to become an extremely important prospect supporting future generations of electronic device that could replace the costly conventional batch processes based on glass substrates that are currently used [11], [12]. It was envisaged to take advantage of its fascinating properties since the optically transparent sheet are foldable, high strength, wide viewing angle and low coefficients of thermal expansion, which is comparable to that of glass and plastics. Furthermore, the higher optical properties of nanocellulose film will give a positive impact towards developing functionalized optical products and lightweight materials for a variety application.

Hence, great efforts have been focused on using cellulose as substrates that have the desired optical properties of paper as electrical conducting sheet and has the potential to be extended to various applications, such as e-book tablets, enewspapers, dynamic wall papers, rewritable maps and learning tools [12]. An important parameter for NCCs is the geometrical axial ratio, which is defined as the length-towidth ratio [13], and is a major factor that controls the properties of NCC based material [6], [8], such as the optical properties of nanocellulose film. It has been shown 
that nanocellulose films that are produced from high axial ratio nanocellulose fibres from wood and softwood appear to be translucent with high porosity [11], [14]-[16] and to obtain higher transparency nanocellulose the film surface are smoothen with several modification process including use of hot pressed [11], [15] and polishing or laminating using transparent resin [11]. According to Nogi et al. (2009) [11], the lack of transparency is caused by surface light scattering, hence if the porosity between the fibres are small enough to avoid light scattering the nanocellulose film will become transparent while maintaining the high performance of the material.

It has also been reported in various studies [14]-[16] that the optical properties of nanocellulose film vary widely depending on the properties of the cellulosic material and preparation conditions of the nanocellulose films.

In this study the use of an alternative NCC source of oil palm empty fruit bunch (OPEFB) for producing nanocellulose films is being investigated. Its optical properties will be controlled via the axial ratio of the NCC namely lower axial ration (LAR), medium axial ratio (MAR) and higher axial ratio (HAR). The OPEFB-NCC was isolated using the 4-acetamido-TEMPO/ $\mathrm{NaBr} / \mathrm{NaClO}$ oxidation system with ultrasonic treatment. Filtration and centrifugation techniques will be used to obtain the NCC with different types of axial ratios. Characterizations of the resulting NCC films were carried out using TEM, uv-vis spectroscopy, SEM, and DSC.

\section{EXPERIMENTAL}

\section{A. Material}

OPEFB fibers obtained from SABUTEK (M) Sdn. Bhd. Teluk Intan, Perak, Malaysia in fibrous strands was used as raw materials for the preparation of OPEFB-pulp. Sodium bromide $(\mathrm{NaBr})$ was obtained from Bendosen, 4-acetamidoTEMPO (2,2,6,6-tetramethylpiperidin-1-oxyl), $98 \%$ was obtained from Sigma-Aldrich, sodium hydroxide $(\mathrm{NaOH})$ was obtained from Fine chemicals, $37 \%$ acid hydrochloric $(\mathrm{HCl})$, was obtained from GRëc, sodium hypochlorite $(\mathrm{NaOCl}) 10 \%$ available chlorine was obtained from $\mathrm{R} \& \mathrm{M}$ Chemicals and all chemicals used as received.

\section{B. Preparation of Bleached Pulp}

OPEFB bleached cellulose pulp was prepared using the environmentally benign process described by Wan Rosli et al. (2003) [5], Leh et al. (2008) [17] and Wan Rosli et al. (2011) [3]. This procedure involves water pre-hydrolysis of the OPEFB strands, followed by soda-anthraquinone pulping. The unbleached pulp was then bleached using a totally chlorine-free (TCF) sequence of oxygen $(\mathrm{O})$, ozone (Z) and peroxide $(\mathrm{P})$.

\section{TEMPO Oxidation}

For the preparation of oxidized OPEFB-pulp the oxidation procedure was carried out based on the procedures of Mishra et al. (2011) [18] and Saito et al. (2007) [19] with minor modification. In brief, OPEFB-pulp (3 g) was suspended in water $(500 \mathrm{~mL})$ containing 4-acetamidoTEMPO (0.048 g) and sodium bromide $(0.48 \mathrm{~g})$. The mixture was then poured into a four-neck flask having a mechanical stirrer, $\mathrm{pH}$ meter and thermocouple and later placed in water bath sonication (model Branson 8510). Then $30 \mathrm{~mL}$ of sodium hypochlorite solution was added dropwise to the cellulose slurry followed by $0.5 \mathrm{M} \mathrm{NaOH}$ under continuous stirring in order to maintain the $\mathrm{pH}$ at $10.0 \pm 0.1$ during the reaction. The reaction was initiated by setting the power of the ultrasonic cleaner at $100 \%$. Circulating cooling water was used to maintain the reaction temperature at $30^{\circ} \mathrm{C}$. After completion of the reaction (4 hours), the $\mathrm{pH}$ of the solution was adjusted to 7 with $0.5 \mathrm{M} \mathrm{HCl}$ and separated by centrifugation using a centrifuge (model Kubota 5100) at $3500 \mathrm{rpm}$. To obtain water-insoluble fraction of oxidized-pulp the precipitate was then dispersed in distilled water and centrifuged for three times to remove superfluous acid, inorganic salt, and TEMPO respectively.

\section{Preparation of OPEFB Nanocellulose Crystal}

The water-insoluble fraction of TEMPO oxidation cellulose was dispersed by water bath sonication for 24 hour, after which the OPEFB-NCC suspension was separated by centrifugally using a centrifuge (model Kubota 5100) at $3500 \mathrm{rpm}$ for 1 hour. The OPEFB-NCC dispersion was then passed through $15-\mu \mathrm{m}$ sintered glass vacuum filters. The OPEFB-NCC was designated as medium axial ratio (MAR$\mathrm{NCC}$ ), then the filtrate of MAR-NCC as low axial ratio (LAR-NCC) and the residue of MAR-NCC as high axial ratio (HAR-NCC).

\section{E. Preparation of Nanocellulose Film}

$10 \mathrm{~mL}$ of $15 \%$ (w/v) NCC suspension was filled into a $5.5 \mathrm{~cm}$ diameter petri dish and allowed to evaporate over 24 hour period at $40{ }^{\circ} \mathrm{C}$ to yield nanocellulose film. The film thickness was control by casting the same amount of portion of film solution on each petri dish.

\section{F. Transmission Electron Microscope}

TEM is used to assess the formation of the fibrils, predominantly by measuring fibril length and width. TEM micrographs were carried out using the transmission electron microscope Phillips CM12 with Docu Version 3.2 image analysis where drops of OPEFB-NCC suspension $(0.01 \% \mathrm{w} / \mathrm{v})$ were deposited on carbon coated electron microscope grids and allowed to dry. Axial ratio is calculated by equation 1

$$
\text { Axial ratio }=\frac{\text { length }}{\text { width }}
$$

\section{G. Scanning Electron Microscope}

Surface morphology of the OPEFB-NCC films were investigated using SEM Oxford INCA 400 model. A thin section of samples were ripped in half and mounted on tape stubs, one-half sample against the stub and the other perpendicular for cross-sectional imaging. The samples were then sputter-coated with gold prior to morphological examination. The operated voltage was $15 \mathrm{kV}$, and the current changed with the vacuum of the observed circumstance.

\section{H. Transparency}

Photograph of NCC films were taken using digital camera SAMSUNG WB250F to evaluate the optical properties of films. Light transmittance through film was measured in a 
UV-VIS spectrophotometer (Thermo Scientific Corporation Evolution 300). Data were collected at room temperature in the visible region (400 to $800 \mathrm{~nm}$ ).

\section{Thermal Analysis}

DSC thermograms were obtained using a Perkin Elmer DSC6 with approximately $10 \mathrm{mg}$ of material in sealed aluminium pans that were heated from ambient temperature to $400{ }^{\circ} \mathrm{C}$ at a heating rate $10{ }^{\circ} \mathrm{C} / \mathrm{min}$ under nitrogen atmosphere.

\section{RESULTS AND DISCUSSION}

Acid hydrolysis (sulfuric acid being the most investigated) has been the primary method for isolating NCCs, however of late, the use of TEMPO (nitroxyl radical 2,2,6,6tetramethylpyperidine-1-oxyl) has gained momentum.

TEMPO oxidation not only helps to generate substantial amount of cellulose pulp negative charge, but also facilitate the fibrillation process during sonication by weakening the adhesion between the cellulose fibrils leading to a decrease in fibre diameter. To obtain uniformity in the NCC dimensions, centrifugation is used after the fibrillation process. Initially, variables such as centrifugation speed and duration was used to isolate NCC with various axial ratios, unfortunately, no significant variations were achieved from this technique. An alternative promising route, which is based on filtration of NCC suspensions through $15-\mu \mathrm{m}$ microfilter was then used to vary the axial ratio of NCC.

In this study, three samples of OPEFB-NCC were prepared from TEMPO-oxidation pulp with different axial ratios. MAR-NCC was obtained directly from the NCC suspensions after centrifugation, whilst HAR-NCC and LAR-NCC were obtained after filtration of MAR-NCC where the filtrate of MAR-NCC was designated as LARNCC and the residue as HAR-NCC.

TEM micrographs of NCCs (Fig. 1) indicate that all of the NCC samples exhibit as rod like fibrils with a long and straight crystals of cellulose [6], [20]. It can also be observed that the HAR-NCC have longer fibril length as compared to that of LAR-NCC, while MAR-NCC, as expected consists of a mixture of both HAR-NCC and LARNCC fibril length. To confirm this observation, the single crystallites dimensions were measured directly from the TEM micrographs and the results presented in Fig. 2 and Fig. 3. Results indicated that the width of the obtained NCCs are in the same range of 3-6 $\mathrm{nm}$ whereas the length differs considerably in the range of $50-450 \mathrm{~nm}, 50-300 \mathrm{~nm}$ and $150-450 \mathrm{~nm}$ for MAR-NCC, LAR-NCC and HAR-NCC respectively (Fig. 2).

Since the NCC width did not vary upon filtration, it can be deduced that the mechanical actions via filtration and centrifugation did not alter the diameter of NCC's, nevertheless these treatments have an effect on the length of the NCC's. Length distributions of NCCs particles indicate that MAR-NCC and HAR-NCC show a normal symmetrical length distribution with an average length of between 151200 and 301-350 nm respectively, whereas the LAR-NCC shows a skewed distribution with much shorter fibril length (Fig. 3). As a consequence of the length variation resulting from the mechanical treatment of filtration, the axial ratio varies accordingly as shown in Table I.

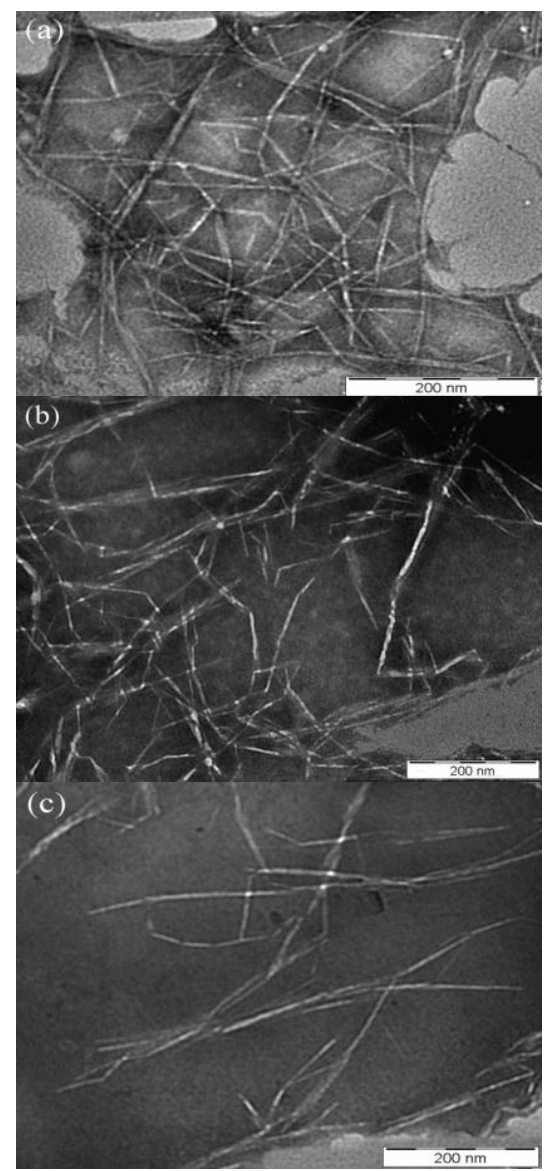

Fig. 1. TEM image of (a) MAR-NCC, (b) LAR-NCC and (C) HAR-NCC.

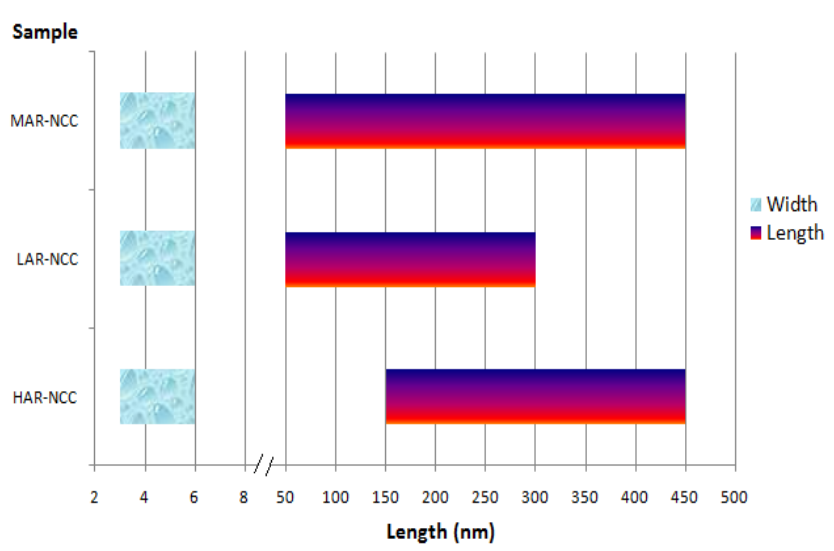

Fig. 2. Dimension range of NCC.

TABLE I: DIMENSION AND AXIAL RATIO OF NCC

\begin{tabular}{cccc}
\hline \multirow{2}{*}{ Sample } & \multicolumn{2}{c}{ midrange } & \\
\cline { 2 - 3 } & width & length & \\
\hline MAR-NCC & 4.5 & 251 & 56 \\
LAR-NCC & 4.5 & 114 & 25 \\
HAR-NCC & 4.5 & 281 & 62 \\
\hline
\end{tabular}

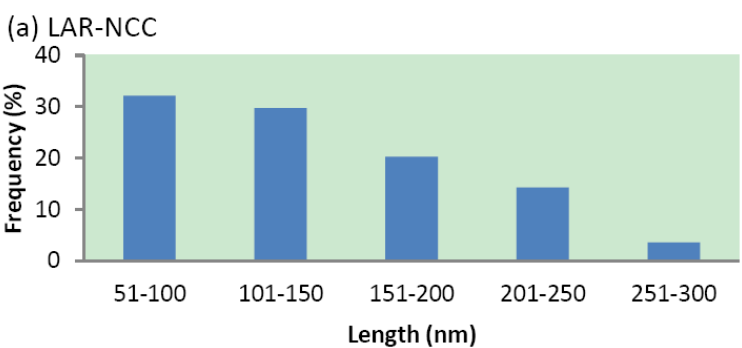



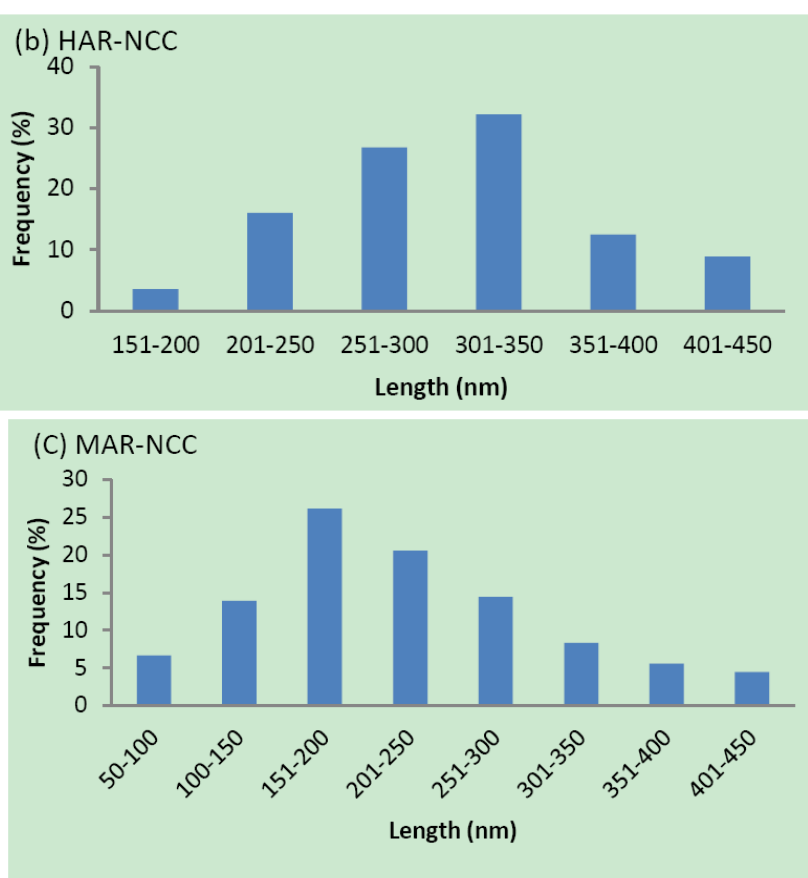

Fig. 3. Length distribution histogram of NCC.

SEM images of the surface morphology for all films appear to be smooth and homogenous (Figs. 4a-c), however the cross section morphology show some differences which is speculated to be related to particle distribution and dispersion since it can be observed from Figs $4 \mathrm{~d}-\mathrm{f}$ that the NCC's are aligned in parallel arrangements and assembled into several layers.

This arrangement is likely to be attributed to the negative charges of the NCC's that were induced by TEMPO oxidation and also orientation of particles might have occurred during film casting or film drying [21]. In addition, it can also be seen that voids (micro-sized pores) are more apparent in the HAR-NCC film compared to that of the MAR-NCC film, whereas it is not noticeable in the LARNCC films. The presence of voids can be related to the length of the NCC particle, since the length of HAR-NCC relatively is longer and as a consequence, the NCC particles cannot occupied the vacancy between the fibril during film casting. However, for MAR-NCC film, voids that are generated from the longer NCC particles were occupied by the shorter NCC particles that act as filler. Consequently, the MAR-NCC film tend to be densely packed approaching to that of LAR-NCC films and comparatively higher than that of HAR-NCC film.

One remarkable feature of nanocellulose film is its high transparency. Visual optical properties of the obtained films are shown in Fig. 5 where they are distinctively different. NCC film of LAR-NCC is optically transparent that had a plastic like appearance where the image (fish) is clearly seen. However, as the axial ratio of NCC increases (HAR-NCC) the visibility is greatly reduced to the point that the objects were no longer visible. This observation is related to light scattering of the particles in the film. With long fibrils of HAR-NCC, there is a significant amount of voids present in the films and due to the difference in the relative index between air (void) and NCC, scattering occurs which give rise to opaque films. On the hand, LAR-NCC because of its short fibrils it tends to fill in the spaces (voids) or it could also be that the interstices between the fibres are small enough to avoid light scattering [11].

Another form of optical property characterization is through measuring light transmittance. It can be seen (Fig 6) that at all wavelengths LAR-NCC and MAR-NCC showed more transmittance than the HAR-NCC, which is in agreement to the visual observation. The light transmittance was approximately $77 \%, 73 \%$ and $53 \%$ for LAR-NCC, MAR-NCC and HAR-NCC films at $600 \mathrm{~nm}$ (visible) wavelength respectively, indicating that the axial ratio of NCC significantly influence the porosity of the film which is associated to the optical transparency of the NCC film.

From these results, it can be concluded that the optical properties of the films could be manipulated by mixing NCC of different axial ratios. The mechanism by which it influences the optical properties is by virtue of the voids that are being generated by these NCC particles. These voids structure in the film increase the probability of light scattering [22] thus reducing the transparency of the films [23] as one move from LAR-NCC to HAR-NCC. With MAR-NCC film since it is densely packed due to the presence of both short and long fibers, this film tend to produce optically transparent film approaching to that of the LAR-NCC film transparency

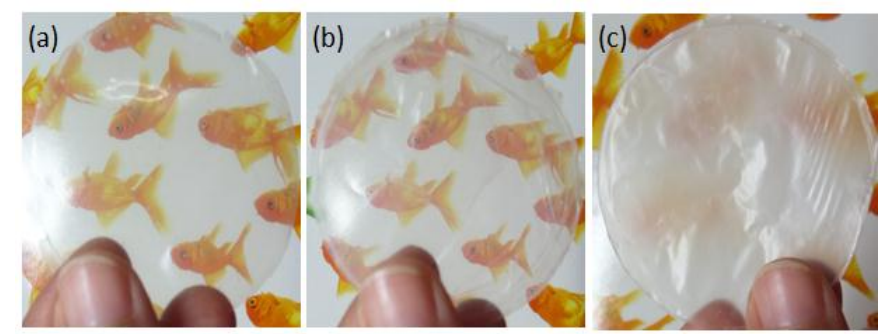

Fig. 5. NCC films of (a) LAR-NCC, (b) MAR-NCC and (c) HAR-NCC.

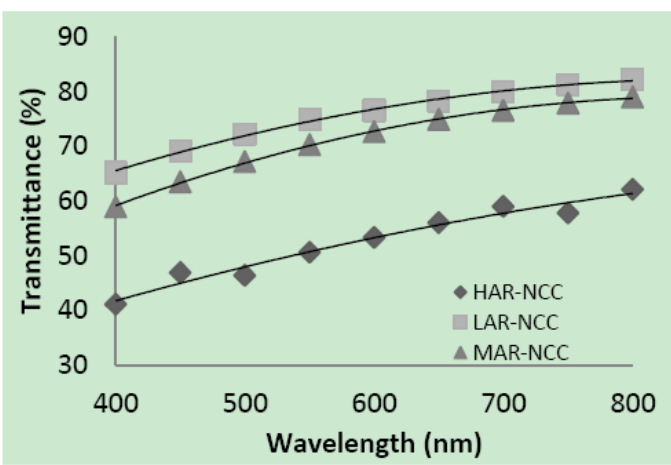

Fig. 6. Optical transparency of NCC films with different axial ratio of NCC.

Thermal stability of cellulose material is among one of the major issue that limits NCC application [24]. To analyse the thermal stability of samples, NCC films were examined using different scanning calorimetric (DSC) in an inert atmosphere. The DSC thermograms (Fig. 7) of the samples show no significant difference between the three samples. The first endothermic peak was observed at $\sim 85^{\circ} \mathrm{C}$ for LARNCC and HAR-NCC, and at $95^{\circ} \mathrm{C}$ for MAR-NCC due to evaporation of adsorbed water in the samples. The water evaporation of sample starts occurring at lower temperature than the boiling point of water. Results also indicate that the evaporation peak of MAR-NCC film increases slightly as compared to that of LAR-NCC and HAR-NCC suggesting 
that MAR-NCC film has higher water holding capability.

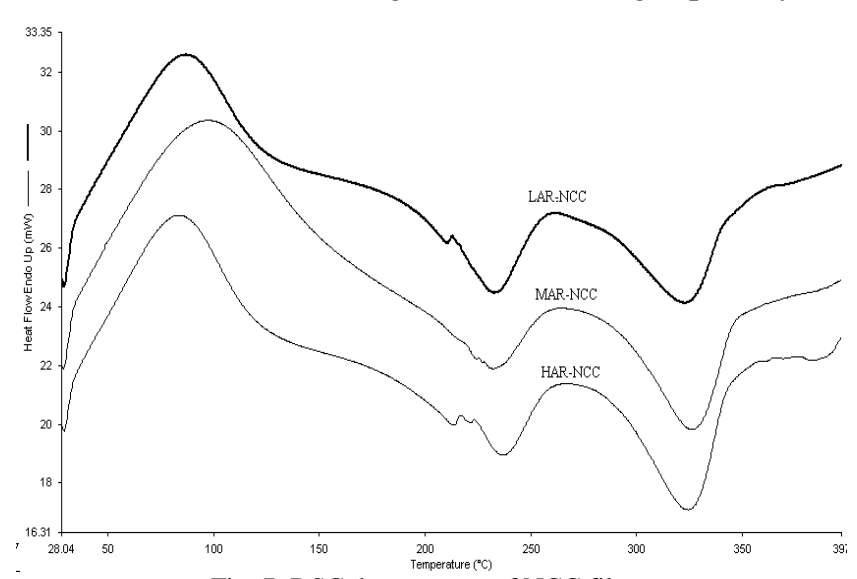

Fig. 7. DSC thermogram of NCC films.

Both exothermic peak at $\sim 235^{\circ} \mathrm{C}$ and $\sim 325^{\circ} \mathrm{C}$ appears to be mainly due to compound decomposition [25, 26]. However closer inspection of these exothermic peaks indicates that the thermal stability of LAR-NCC is slightly lower by $3-4{ }^{\circ} \mathrm{C}$ compared to that of HAR-NCC and MARNCC (TABLE II). This is likely due to the difference in the length of NCC's where it is reported that in general the thermal stability of a polymer increases with an increase in the polymer chain [26].

\begin{tabular}{cccc} 
& \multicolumn{4}{c}{ TABLE II: THERMAL STABILITY OF OPEFB-NCC } \\
\hline & $\begin{array}{c}\text { Water adsorption } \\
\left({ }^{\circ} \mathrm{C}\right)\end{array}$ & $\begin{array}{c}1^{\text {st }} \text { decomposition } \\
\text { peak }\left({ }^{\circ} \mathrm{C}\right)\end{array}$ & $\begin{array}{c}2^{\text {nd }} \text { decomposition } \\
\text { peak }\left({ }^{\circ} \mathrm{C}\right)\end{array}$ \\
\cline { 2 - 4 } MAR-NCC & 95 & 232 & 325 \\
HAR-NCC & 83 & 237 & 325 \\
LAR-NCC & 85 & 233 & 322 \\
\hline
\end{tabular}

\section{CONCLUSION}

NCC's isolated from OPEFB via the TEMPO-oxidation process has a typical width in the range of $6-3 \mathrm{~nm}$ irrespective of the axial ratio differences (HAR-NCC, MAR-NCC and LAR-NCC) and exhibit as long and straight crystals. SEM micrographs show that the surface morphology of films appears to be smooth and homogenous with multilayered deposition of particles, whereas the cross section morphology shows that voids are apparent in the HAR-NCC film and almost none were visible in the LARNCC and MAR-NCC films. LAR-NCC and MAR-NCC films are optically transparent whilst the HAR-NCC films are opaque in nature. This visual observation is in agreement with the light transmittance measurement where higher transmittances were observed in the LAR-NCC and MARNCC film as compared to HAR-NCC film in the visible wavelength section. These results indicate that the axial ratios of NCC can affect the optical properties of NCC films by virtue of the presence of voids in films. Thermal analyses show that there are no significant differences in thermal stability observed even though the axial ratios of samples are different. However, results indicate that MAR-NCC film has higher water holding capability in comparison to that of LAR-NCC and MAR-NCC.

Results from this study suggest that geometrical axial ratio, i.e. length to diameter (L/D) is an important parameter in controlling the properties of nanocellulose films by virtue of the presence of voids. One of the most obvious prospect of exploiting this finding is in the preparation of porous templates where a high geometrical aspect ratio NCC's can be used to produce a low density and highly porous lightweight aerogels that have the potentials to be used in a variety of applications such as thermal and acoustic insulation, as a catalyst or a catalyst carrier or as thickening agents in paints and cosmetics.

\section{REFERENCES}

[1] P. Q. N. Wendy, H. L. Lam, F. Y. Ng, M. Kamal, and J. H. E. Lim, "Waste-to-wealth: green potential from palm biomass in Malaysia," Journal of Cleaner Production, vol. 34, pp. 57-65, 2012.

[2] A. H. R. Aljuboori, "Oil palm biomass residue in malaysia: availability and sustainability," International Journal of Biomass and renewable, vol. 2, no. 1, 2013

[3] W. D. Wanrosli, R. Rohaizu, and A. Ghazali, "Synthesis and characterization of cellulose phosphate from oil palm empty fruit bunches microcrystalline cellulose," Carbohydrate Polymers, vol. 84 , no. 1 , pp. 262-267, 2011

[4] W. D. Wanrosli, Z. Zainuddin, and L. K. Lee, "Influence of pulping variables on the properties of Elaeis guineensis soda pulp as evaluated by response surface methodology," Wood Science and Technology, vol. 38, no. 3, pp. 191-205, 2004.

[5] W. D. Wan Rosli, C. P. Leh, Z. Zainuddin, and R. Tanaka, "Optimisation of soda pulping variables for preparation of dissolving pulps from oil palm fibre," Holzforschung, vol. 57, no. 1, pp. 106-113, 2003.

[6] K. Fleming, D. G. Gray, and S. Matthews, "Cellulose crystallites," Chemistry-a European Journal, vol. 7, no. 9, pp. 1831-1835, 2001.

[7] D. Klemm, F. Kramer, S. Moritz, T. Lindstrom, M. Ankerfors, D Gray, and A. Dorris, "Nanocelluloses: A new family of nature-based materials," Angewandte Chemie-International Edition, vol. 50, no. 24, pp. 5438-5466, 2011.

[8] B. L. Peng, N. Dhar, H. L. Liu, and K. C. Tam, "Chemistry and applications of nanocrystalline cellulose and its derivatives: a nanotechnology perspective," Canadian Journal of Chemical Engineering, vol. 89, no. 5, pp. 1191-1206, 2011.

[9] W. Hamad, "On the development and applications of cellulosic nanofibrillar and nanocrystalline materials," Canadian Journal of Chemical Engineering, vol. 84, no. 5, pp. 513-519, 2006.

[10] Y. Habibi, L. A. Lucia, and O. J. Rojas, "Cellulose nanocrystals: chemistry, self-assembly, and applications," Chemical Reviews, vol. 110, no. 6, pp. 3479-3500, 2010.

[11] M. Nogi, S. Iwamoto, A. N. Nakagaito, and H. Yano, "Optically transparent nanofiber paper," Advanced Materials, vol. 21, no. 16, pp. 1595-+, 2009.

[12] J. Shah and R. M. Brown, "Towards electronic paper displays made from microbial cellulose," Applied Microbiology and Biotechnology, vol. 66 , no. 4 , pp. $352-355,2005$

[13] D. Tatsumi, S. Ishioka, and T. Matsumoto, "Effect of fiber concentration and axial ratio on the rheological properties of cellulose fiber suspensions," Journal of the Society of Rheology Japan, vol. 30 , no. 1, pp. 27-32, 2002.

[14] H. Sehaqui et al., "Strong and tough cellulose nanopaper with high specific surface area and porosity," Biomacromolecules, vol. 12, no. 10, pp. 3638-3644, 2011

[15] H. Y. Wang, D. G. Li, and R. R. Zhang, "Preparation of ultralong cellulose nanofibers and optically transparent nanopapers derived from waste corrugated paper pulp," Bioresources, vol. 8, no. 1, pp. 1374-1384, 2013.

[16] Q. Q. Wang, J. Y. Zhu, and J. M. Considine, "Strong and optically transparent films prepared using cellulosic solid residue recovered from cellulose nanocrystals production waste stream," Acs Applied Materials \& Interfaces, vol. 5, no. 7, pp. 2527-2534, 2013.

[17] C. P. Leh, W. D. W. Rosli, Z. Zainuddin, and R. Tanaka, "Optimisation of oxygen delignification in production of totally chlorine-free cellulose pulps from oil palm empty fruit bunch fibre," Industrial Crops and Products, vol. 28, no. 3, pp. 260-267, 2008.

[18] S. P. Mishra, J. Thirree, A. S. Manent, B. Chabot, and C. Daneault, "Ultrasound-catalyzed tempo-mediated oxidation of native cellulose for the production of nanocellulose: effect of process variables," Bioresources, vol. 6, no. 1, pp. 121-143, 2011. 
[19] T. Saito, S. Kimura, Y. Nishiyama, and A. Isogai, "Cellulose nanofibers prepared by TEMPO-mediated oxidation of native cellulose," Biomacromolecules, vol. 8, no. 8, pp. 2485-2491, 2007.

[20] S.J. Eichhorn et al., "Review: current international research into cellulose nanofibres and nanocomposites," Journal of Materials Science, vol. 45, no. 1, pp. 1-33, 2010.

[21] B. L. Holt, S. D. Stoyanov, E. Pelan, and V. N. Paunov, "Novel anisotropic materials from functionalised colloidal cellulose and cellulose derivatives," Journal of Materials Chemistry, vol. 20, no. 45, pp. 10058-10070, 2010.

[22] V. Džimbeg-Malčić, Ž. Barbarić-Mikočević, and K. Itrić, "Kubelkamunk theory in describing optical properties of paper technical gazette," Tehnicki Vjesnik-Technical Gazette, vol. 18, no. 1, pp. 117 124, 2011.

[23] Z. Q. Fang et al., "Highly transparent and writable wood all-cellulose hybrid nanostructured paper," Journal of Materials Chemistry C, vol 1, no. 39, pp. 6191-6197, 2013.

[24] L. Brinchi, F. Cotana, E. Fortunati, and J. M. Kenny, "Production of nanocrystalline cellulose from lignocellulosic biomass: Technology and applications," Carbohydrate Polymers, vol. 94, no. 1, pp. 154$169,2013$.

[25] J. Scheirs, G. Camino, and W. Tumiatti, "Overview of water evolution during the thermal degradation of cellulose," European Polymer Journal, vol. 37, no. 5, pp. 933-942, 2001.
[26] C. L. Beyler and M. M. Hirschler, "Thermal Decomposition of Polymers." in SFPE Handbook of Fire Protection Engineering, 3th ed., Philip J. DiNenno et al., Eds. Quincy, Massachusetts 02269: National Fire Protection Association, pp. 110-131, 2002.

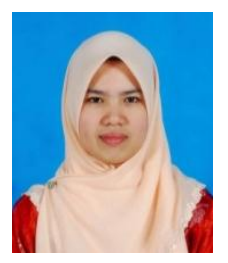

Rohaizu Roslan graduated with a B.Sc. in industrial chemistry and subsequently received her master's degree in bioresources, paper and coating technology from Universiti Sains Malaysia in 2011. She is currently pursuing a $\mathrm{PhD}$ at the School of Industrial Technology, Universiti Sains Malaysia under the supervision of Prof. Wan Rosli Wan Daud. Her research interests include sustainable production of materials from oil palm empty fruit bunch (OPEFB) biomass, production of cellulose and its derivatives and isolation of nanocellulose from OPEFB.

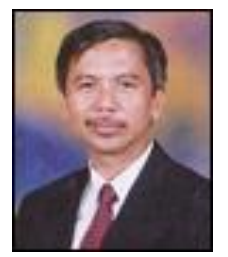

Wan Rosli Wan Daud is a professor at the School of Industrial Technology, Universiti Sains Malaysia. His research centers on the use of biomass in particular the oil palm fibers as materials for the production of cellulose (including nanocelluloses) and its modifications via various chemical and mechanical treatments. 